\title{
Prospecção de Artigos e Patentes sobre Plantas Medicinais Presentes na Caatinga Brasileira
}

\author{
Prospecting Articles and Patents on Medicinal Plants Present in the \\ Brazilian Caatinga
}

\author{
Sannyele Alcantara Emiliano ${ }^{1}$ \\ Tatiane Luciano Balliano ${ }^{1}$ \\ ${ }^{1}$ Universidade Federal de Alagoas, Instituto de Química e Biotecnologia, Maceió, AL, Brasil
}

\begin{abstract}
Resumo
Plantas medicinais possuem princípios ativos que ajudam no tratamento de doenças e podem levar à cura. São utilizadas por meio de chás ou infusões, que devem ser ingeridos enquanto durar o tratamento. Ainda hoje, nas regiões mais pobres do país, estas plantas vêm sendo comercializadas em feiras livres, mercados, sendo ainda encontradas em quintais para seu uso fitoterápico. Entretanto, na última década as plantas medicinais têm se destacado como produto de valor agregado, onde o número de países que importam essa matéria-prima vem se ampliando, compreendendo países como Alemanha, Estados Unidos, Singapura, Malásia e Japão, estes os maiores importadores de plantas medicinais e aromáticas. Assim, este trabalho refere-se a um estudo prospectivo de artigos e patentes relacionadas a espécies de plantas de interesse medicinal, onde são verificados alguns indicadores que demonstram a importância do conhecimento da utilização das mesmas, bem como o interesse de mercado.
\end{abstract}

Palavras-chave: Plantas nativas da caatinga. Princípios ativos. Fitoterápicos.

\begin{abstract}
Medicinal plants have active principles that help in the treatment of diseases and can lead to healing. They are used by means of teas or infusions that must be ingested during the duration of the treatment. Even today, in the poorest regions of the country, these plants are being marketed in open markets, markets, and are still found in quintals for their phytotherapeutic use. In the last decade, however, medicinal plants have become a valuable product, with countries such as Germany, the United States, Singapore, Malaysia and Japan being the largest importers of medicinal and aromatic plants. Thus, this work refers to a prospective study of articles and patents related to plant species of medicinal interest, where are verified some indicators that demonstrate the importance of knowledge of the use of them as well as market interest.
\end{abstract}

Keywords: Plants native to the caatinga. Active principles. Phytotherapics. 


\section{Introdução}

O uso de plantas medicinais no tratamento e na cura de enfermidades é bastante antigo. $\mathrm{Na}$ caatinga nordestina estas plantas são amplamente utilizadas na medicina popular pelas comunidades locais (CORRÊA; LIN; SCHEFFER, 1991). É possível constatar o interesse por plantas medicinais tanto em âmbito nacional quanto internacional, por elas apresentarem um potencial terapêutico e econômico, visado, especialmente, pela indústria farmacêutica (MAIOLI-AZEVEDO; FONSECA-KRUEL, 2007).

A utilização segura desses fitoterápicos está vinculada ao conhecimento adequado, de forma a não causar perda na efetividade dos princípios ativos existentes nas plantas, bem como evitar o risco de intoxicações geradas pelo uso indevido (ARNOUS; SANTOS; BEINNER, 2005) . Portanto, faz-se necessário validar as plantas medicinais como medicamentos eficazes e seguros, de modo que a autorização oficial de seu uso seja fundamentada em evidências experimentais comprobatórias de que o risco a que se expõem aqueles que as utilizam seja suplantado pelos benefícios que possam delas advir (SIMÕES; SCHENKEL; GOSMANN, 2002).

A realização de estudos etnobotânicos e etnofarmacológicos pode proporcionar diversos avanços na ciência farmacêutica e na descoberta de novos fármacos, além de promover a preservação da biodiversidade, sendo importante na investigação do uso das plantas locais combinadas a fármacos já conhecidos e outras tecnologias biomédicas, bem como respeitando o conhecimento das comunidades locais (ALBUQUERQUE, HANAZAKI, 2006).

A caatinga é um bioma exclusivamente brasileiro e, apesar desta exclusividade, ainda não foi explorada o suficiente para direcionar os estudos botânicos nesta área. São 341 espécies no Nordeste utilizadas como medicinais, sendo 34 prioritariamente da Caatinga (SAMPAIO et al.,2006). Nesse sentido, tem-se a crescente busca pelo conhecimento das espécies nativas com potencial medicinal (OMS, 1978), sendo de interesse deste trabalho: Alecrim de Vaqueiro (Lippiaaffgracilis), Feijão Bravo (Capparis flexuosa), Imburana (Amburana cearenses), Sacatinga Branca (Crotonargyrophyllus) e Velame (Crotonheliotropiifolius).

Essas considerações justificam o interesse em desenvolver uma revisão sobre o uso de plantas medicinais da caatinga como recurso terapêutico, para a interpretação do conhecimento produzido na área e com o propósito de auxiliar no desenvolvimento de futuras investigações. Sendo assim, o presente estudo teve como objetivo analisar a importância relativa das espécies coletadas no município de Delmiro Golveia - AL, visando a identificar a sua potencialidade frente a algumas enfermidades.

\section{Metodologia}

A pesquisa fitoquímica é importante, principalmente, quando ainda não são dispostos todos os estudos químicos com espécies de interesse popular, tendo como objetivo conhecer os compostos químicos das espécies vegetais e avaliar sua presença nos mesmos, identificando-se grupos de metabólitos secundários relevantes (SIMÕES et al., 2010) .

As cinco espécies estudadas foram selecionadas por serem plantas medicinais nativas do bioma Caatinga, presentes no município de Delmiro Gouveia, cujos usos e indicações foram 
mais citados pela comunidade da região. As folhas e caules das espécies foram coletadas em maio de 2017, de acordo com a época mais frutífera das espécies.

Para o artigo foram realizadas buscas nas bases de dados Web of Science, Scopus, Science Direct, e Periódicos Capes, com as palavras-chaves analisadas nos resumos presentes na Tabela 1.

No presente trabalho, para a busca patentária, utilizaram-se as bases de dados do Lens, o Instituto Nacional de Propriedade Intelectual (INPI), como referência nacional, o Espacenet, que busca patentes do mundo todo, a WIPO, que dispõe da proteção da propriedade intelectual ao redor do mundo através da cooperação entre estados, e o QuestelOrbit, ferramenta que, além das informações coletadas, permite a geração e visualização de gráficos sobre o conjunto de patentes, empresas depositantes e inventores.

\section{Resultados e Discussão}

Os estudos realizados com plantas da caatinga estão relacionados à potencialidade farmacológica que as mesmas possuem, à composição química das mesmas, bem como a alternativas para conservação das espécies no seu habitat natural. Na busca, foram encontrados artigos entre os anos de 1983 a 2018.

Tabela 1 - Escopo da Busca de Artigos

\begin{tabular}{ccccc} 
Palavras- chave & $\begin{array}{c}\text { Web OF } \\
\text { SCIENCE }\end{array}$ & $\begin{array}{c}\text { ScIence } \\
\text { Direct }\end{array}$ & $\begin{array}{c}\text { Periódico } \\
\text { CAPES }\end{array}$ & Scopus \\
Caatinga Plants & 83 & 103 & 221 & 786 \\
plantssemi-aridregionofnortheasternBrazil & 5 & 33 & 16 & 105 \\
Lippiaaffgracilis & 2 & 19 & 2 & 2 \\
Capparis flexuosa & 9 & 5 & 22 & 29 \\
Amburana cearensis & 64 & 19 & 37 & 134 \\
Crotonargyrophyllus & 7 & 2 & 12 & 11 \\
Crotonheliotropiifolius & 5 & 4 & 10 & 20 \\
\hline
\end{tabular}

Fonte: Elaborada pelas autoras deste artigo (2018)

Os artigos encontrados para os termos "caatinga Plants" e "plantssemi-aridregionofnortheasternBrazil" mostram o interesse no potencial medicinal destas plantas a fim de combater doenças que assolam todo o mundo, principalmente o Brasil, como a epidemia causada pelo Staphylococcus. O estudo destas plantas endêmicas da caatinga teve um crescimento significativo nas publicações a partir de 2007, quando pesquisas promovendo o uso sustentável da biodiversidade de plantas foram incentivadas, tendo em vista que algumas espécies vêm sendo utilizadas por boa parte da população como medicamentos. Para a validação dessas espécies como fitoterápicos é preciso confirmar suas propriedades farmacológicas e a ausência de toxicidade, justificando assim os investimentos para pesquisas na área (Figura 1). 
Figura 1 - Documentos obtidos entre 1939 e 2018 para os termos "Caatinga Plants" e "plantssemi-aridregionofnortheasternBrazil"

\section{Documents by year}
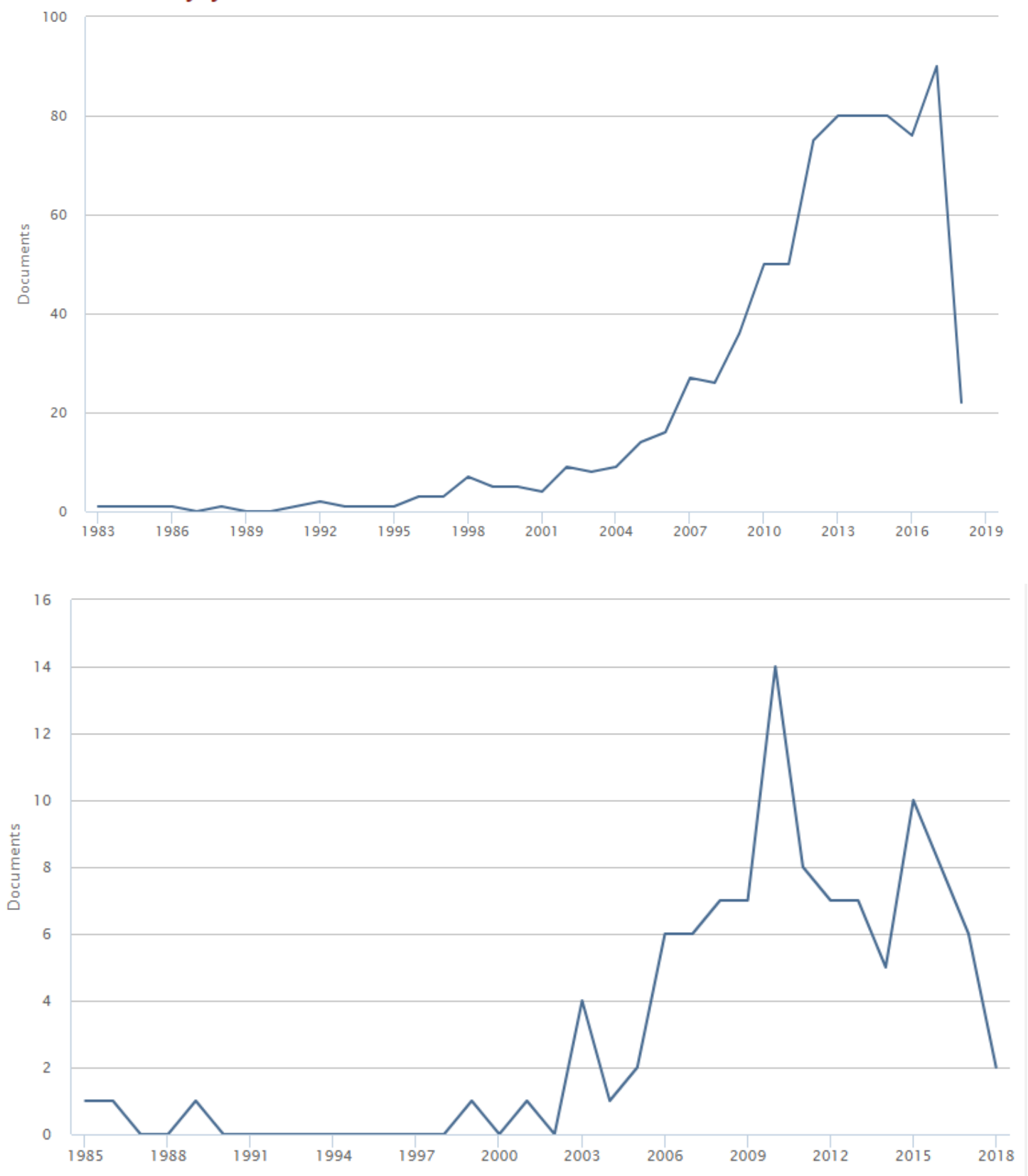

Fonte: Scopus (2018)

Para o requerido tema, de 1983 a 2018, o Brasil supera todos os países em termos de publicações (Figura 2). O país lidera a produção de artigos científicos em periódicos, tendo publicado 747 trabalhos dos 786 encontrados referentes a plantas nativas da caatinga e, mais especificamente, 98 de 105 relacionados às buscas de plantas do Nordeste brasileiro. Os estudos etnofarmacológico/etnobotânico agregam conhecimento popular com pesquisa, de forma que as plantas medicinais podem ser estudadas e, após o conhecimento do efeito fitoterápico, utilizadas para fabricação de novos medicamentos, apresentando potencial de contribuir para o sistema local de saúde e seus usuários. O grande número de publicações brasileiras deve-se à vasta acessibilidade ao objeto de estudo. 
Figura 2 - Documentos classificados por país de publicação para os termos "Caatinga Plants" e "plantssemi-aridregionofnortheasternBrazil"
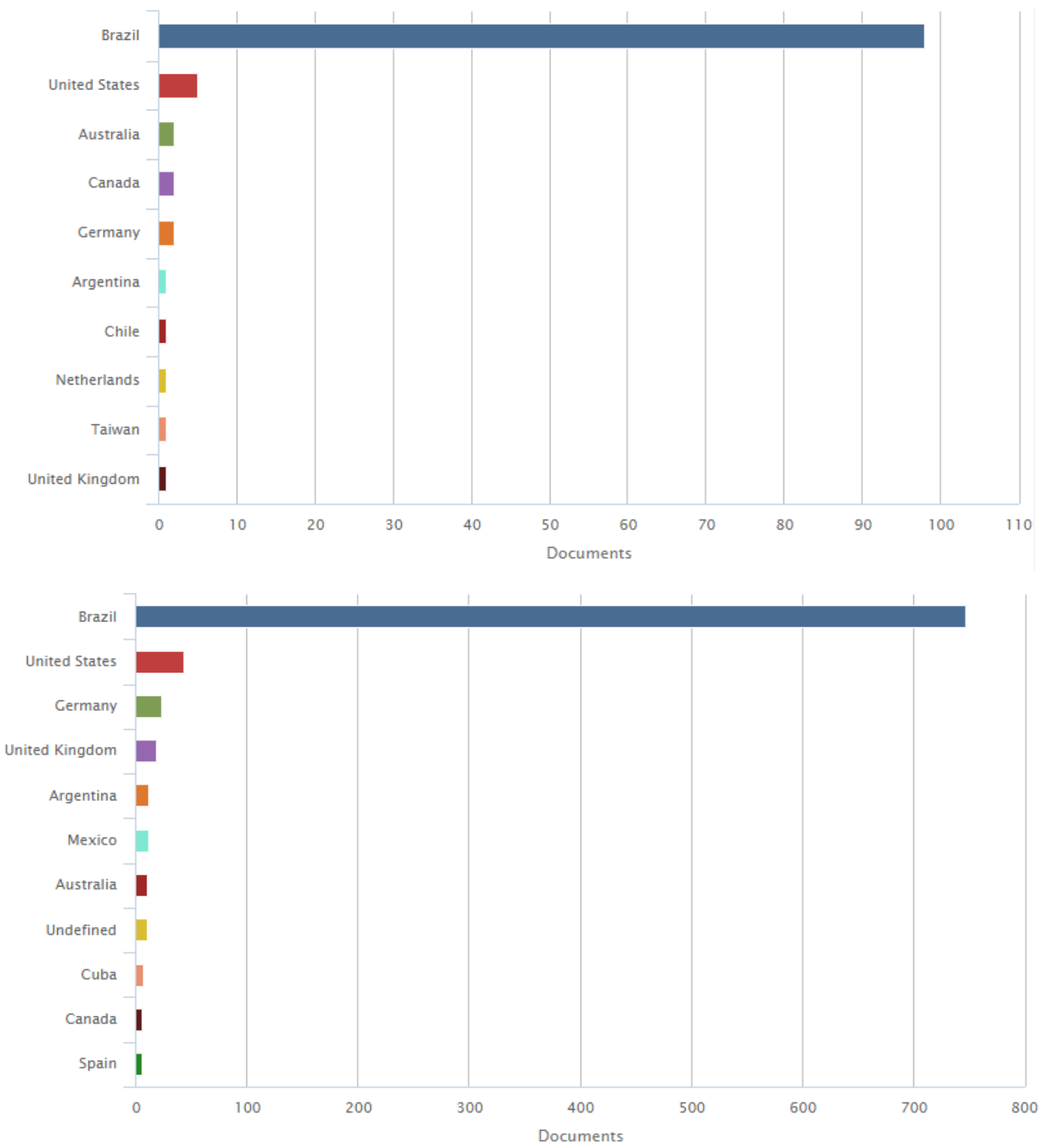

Fonte: Scopus (2018)

Para a espécie Lippiaaffgracilisos, artigos mostram a química, a farmacologia e os seus tradicionais usos, como antibactericida e inseticida, para algumas espécies de plantas. Em contrapartida, alguns gêneros possuem longos estudos para as famílias citadas, mas o conhecimento da espécie em análise continua muito pequeno, o que intriga os pesquisadores a descobrirem mais, como é o caso da Capparis, comum na região da Jamaica, sendo a Capparis flexuosa encontrada especialmente no Nordeste do Brasil, onde vem sendo realizados estudos para predição de suas propriedades farmacológicas, fato ainda não determinado. Quanto à espécie Crotonargyrophyllus, encontrou-se um artigo descrevendo seu potencial anti-inflamatório, enquanto que a Crotonheliotropiifolius, da mesma família, apresentou potencial antibacteriano, antifúngico e antibiótico em seu óleo essencial. 
Figura 3 - Áreas de estudo Capparis flexuosa

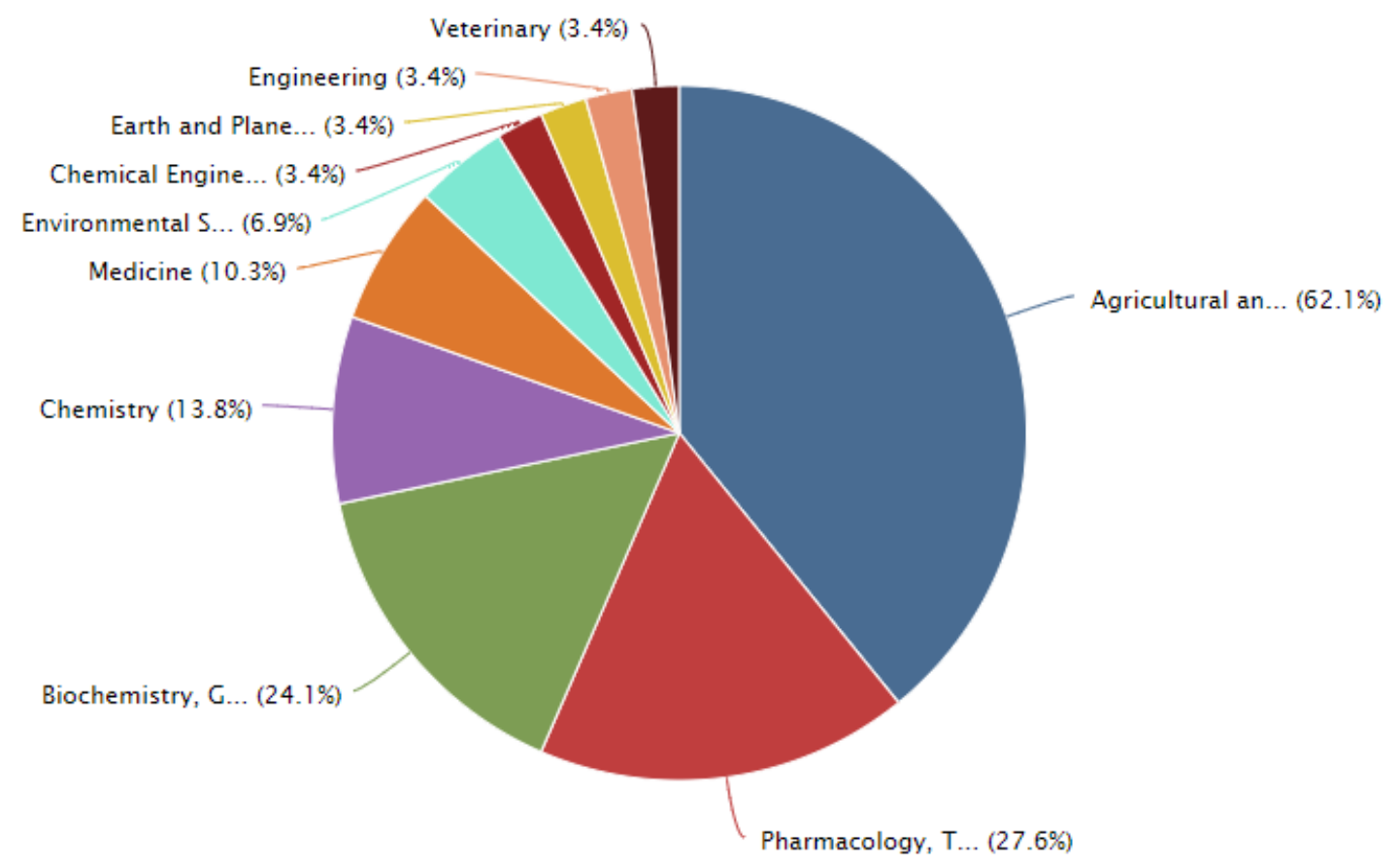

Fonte: Scopus, 2018

Figura 4 - Áreas de pesquisa Amburana Cearensis

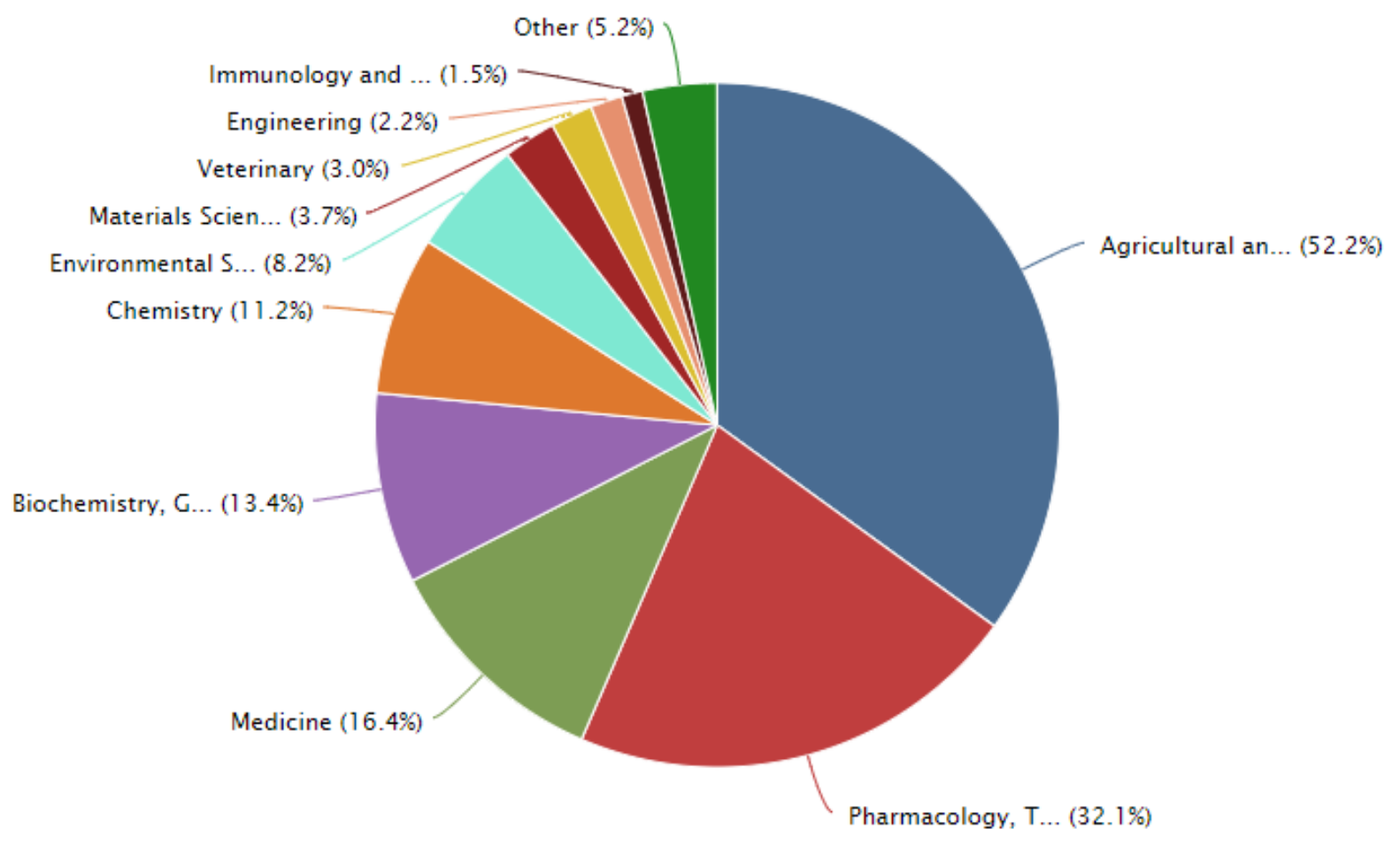

Fonte: Scopus (2018) 
Figura 5 - Áreas de pesquisa Crotonargyrophyllus

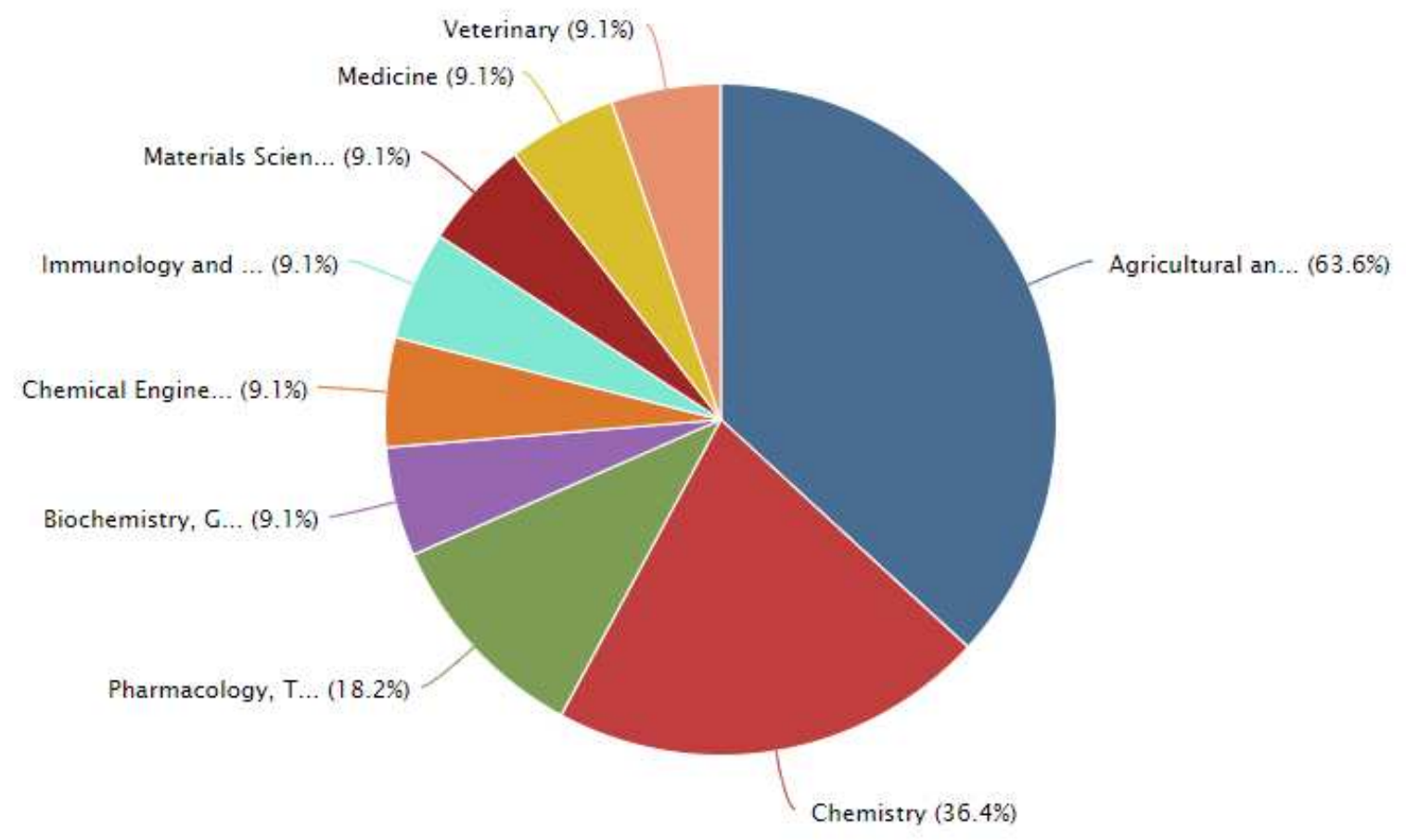

Fonte: Scopus (2018)

Figura 6 - Áreas de pesquisa Crotonheliotropiifolius

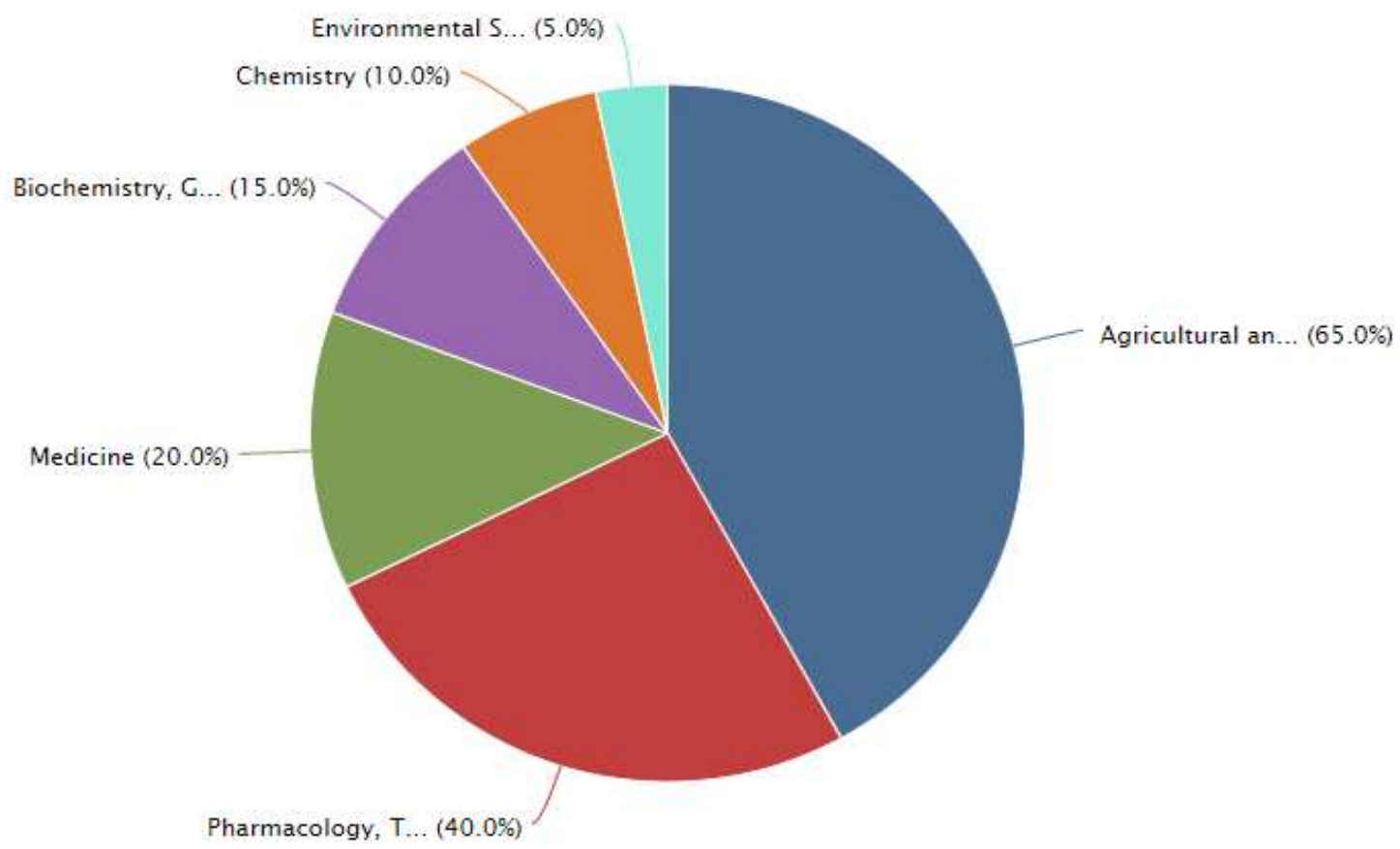

Fonte: Scopus (2018)

Percebe-se que, para todas as espécies analisadas, suas áreas de pesquisa se assemelham, destacando-se o setor da agricultura, na qual suas publicações estão focadas na fenologia das plantas, bem como o teor de nutrientes presentes em seus tecidos. A composição química de cada um dos gêneros examinados é o que diferencia as atividades biológicas e farmacológicas de cada espécie (Figuras 3, 4, 5 e 6). 
Atualmente, com o crescimento no desenvolvimento científico, acompanhado da expansão tecnológica, deu-se importância à proteção da propriedade intelectual como mecanismo da garantia de direitos e estímulos ao investimento através de patentes. Buscaram-se as patentes que contivessem as palavras-chave demonstradas na Tabela 2 no título e no resumo.

Tabela 2 - Escopo de busca de Patentes

\begin{tabular}{cccccc} 
PalavRAS-CHAVE & INPI & LENS & EsPaCENET & WIPO & QuestelORBIT \\
Medicinal Plants & 4.101 & 24.626 & 2.906 & 4.409 & 3.269 \\
Caatinga andPlants & 0 & 0 & 0 & 15 & 1 \\
PlantsandBrazilian & 0 & 0 & 0 & 138 & 29 \\
Lippiagracilis & 4 & 0 & 0 & 6 & 6 \\
Capparis flexuosa & 0 & 0 & 0 & 0 & 0 \\
Amburana cearenses & 0 & 0 & 0 & 0 & 2 \\
Crotonargyrophyllus & 1 & 0 & 0 & 1 & 0 \\
Crotonheliotropiifolius & 0 & 0 & 0 & 0 & 0 \\
\hline
\end{tabular}

Fonte: Elaborada pelas autoras deste artigo (2018)

As patentes referenciadas a plantas brasileiras atingem a marca de 3.269 depósitos ao redor do mundo, destacando-se na criação de inseticidas inéditos, a partir da combinação de proteínas, ou no uso do fungo Lewiachlamidosporiformans como biológico herbicida para o controle de famosas ervas daninhas. Ademais, sabe-se que a flora brasileira se destaca quanto à sua biodiversidade, apresentando cenário propício para a pesquisa, tendo em vista a gama de plantas que auxiliam no tratamento de sérias doenças, bem como do Lupus Eritematoso ou da AIDS. Outra proposta e invenção alcançada foi de um polímero natural em forma de um filme capaz de curar, bem como anestesiar, a área aplicada. Das várias áreas de invenção relacionadas ao uso de plantas nativas do Brasil, a farmacologia, destacam-se, mais precisamente, aquelas oriundas do Nordeste brasileiro, sendo este bioma o habitat natural para as espécies estudadas.

Dentre as patentes encontradas para a espécie de planta do Gênero Lippia, destacam-se produtos com base ativa no controle de praga na agricultura, no uso tópico humano, como eliminação de dermatocitoses. Além de anestésico, ela apresenta ainda atividade antimicrobiana e cicatrizante, atividade carrapaticida e larvicida, inclusive frente ao Aedes Aegypti. Já quanto à espécie Amburana cearenses, uma patente foi encontrada relativa às suas atividades anti-inflamatórias e broncodilatadoras e outra quanto ao processo de desenvolvimento de um isolado protéico a partir de sementes de cumaru ou imburana-de-cheiro para ser utilizado como suplemento na alimentação humana. A espécie Crotonargyrophyllus destaca-se no uso do óleo essencial de suas folhas como agente anti-inflamatório, enquanto a Crotonheliotropiifolius não apresentou patentes depositadas.

Com o Sistema QuestelOrbit, algumas análises estatísticas foram possíveis na visualização de informações gerais sobre as patentes encontradas quanto ao uso de Plantas Brasileiras. A Figura 7 apresenta os maiores depositantes nesta área, que coincidem com os depositantes que permanecem no topo mesmo ao se considerar as patentes expiradas. 
Figura 7 - Ranking de depositantes

Top technology players

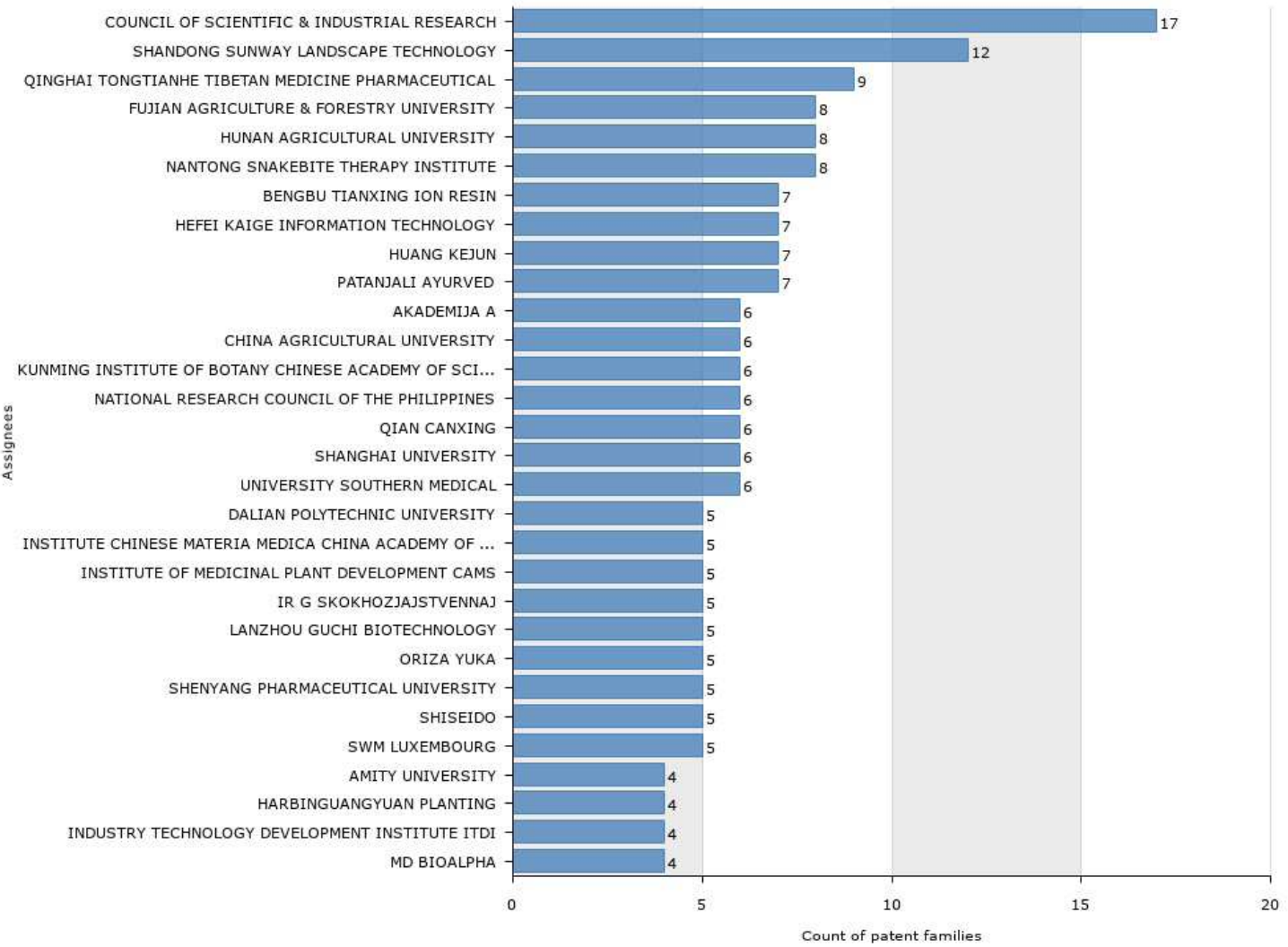

Fonte: Questel, Orbit (2018)

Figura 8 - Depositantes com patentes expiradas

Players dead \& alive Patents

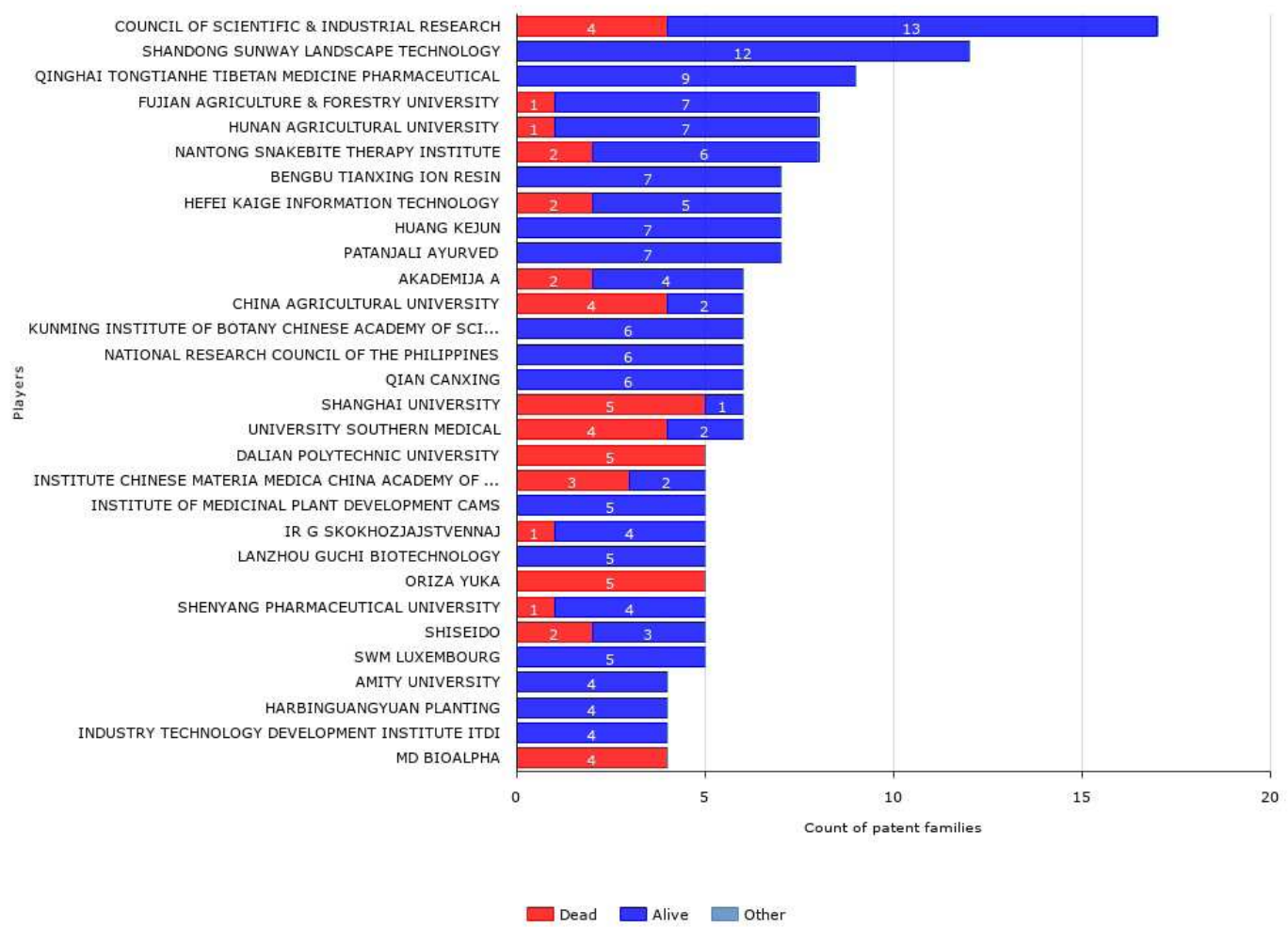

Fonte: Questel, Orbit (2018) 
A busca permitiu ainda reconhecer a dinâmica de inventividade do escopo estudado, apresentando diferentes perfis que estão em construção de sua carreira inventiva ou aqueles já há muito estabilizados. Estes perfis então dependem da estratégia implementada pelo requerente. Por exemplo, um setor com crescimento linear representa uma entidade com interesse contínuo no campo de estudo, enquanto que um setor com crescimento exponencial é indicativo de uma corrida por uma patente. Em contrapartida, quando o número de patentes diminui, isso é sinal do desligamento dos principais depositantes no campo. Ao considerar os anos decorridos, o avanço tecnológico apresentou-se de forma crescente. Entretanto, em comparação aos anos anteriores, 2018 não vem mostrando bons resultados, mesmo ao se considerar somente o primeiro semestre; desta maneira, para o segundo espera-se um avanço tecnológico. Tal comportamento pode ser visto na Figura 9.

Figura 9 - Tendência de investimento na tecnologia

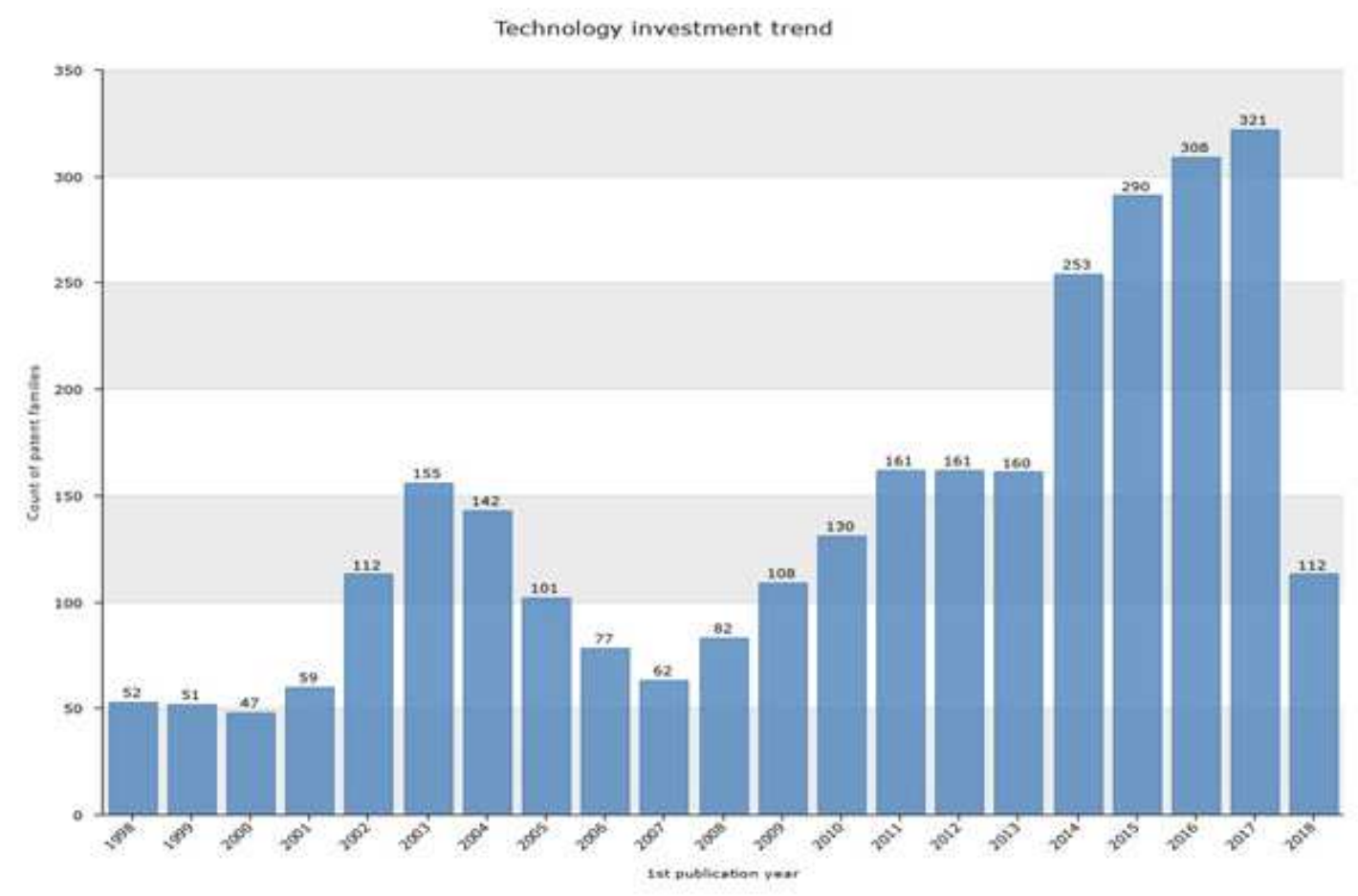

Fonte: Questel, Orbit (2018)

No geral, no âmbito de inovação tecnológica a competitividade é a capacidade de uma empresa ou núcleo de pesquisa se tornarem mais produtivos e lucrativos dentro do seu universo, na qual os pesquisadores devem tomar as melhores decisões para alcançar os resultados mais adequados que serão indicados no montante de depósitos de patentes realizados em um determinado período. O número de patentes solicitadas por cada país é um bom indicativo do nível de inovação e do andamento da economia. Desta maneira, como pode ser visto na Figura 7, os pesquisadores e empresas chinesas destacam-se no depósito de patentes, sendo seguidos pelos norte-americanos, fato que se relaciona ainda ao domínio do mercado mundial dessas nações. Este domínio é decorrente da estratégia do solicitante, que registra o pedido da patente em regiões locais a fim de manter o controle.

Como explicação para o destaque da nação chinesa neste setor, tem-se a medicina tradicional Chinesa, com origem há 4 mil anos, destacando-se no uso de plantas medicinais através 
de infusões, chás, remédios naturais, dentre outros. Tendo em vista a biodiversidade da flora brasileira, o território nacional, torna-se um vasto campo de pesquisa para essa civilização.

Figura 10 - (a) Localização de patentes depositadas e (b) mercados dos concorrentes RSD location (without EP and wo)

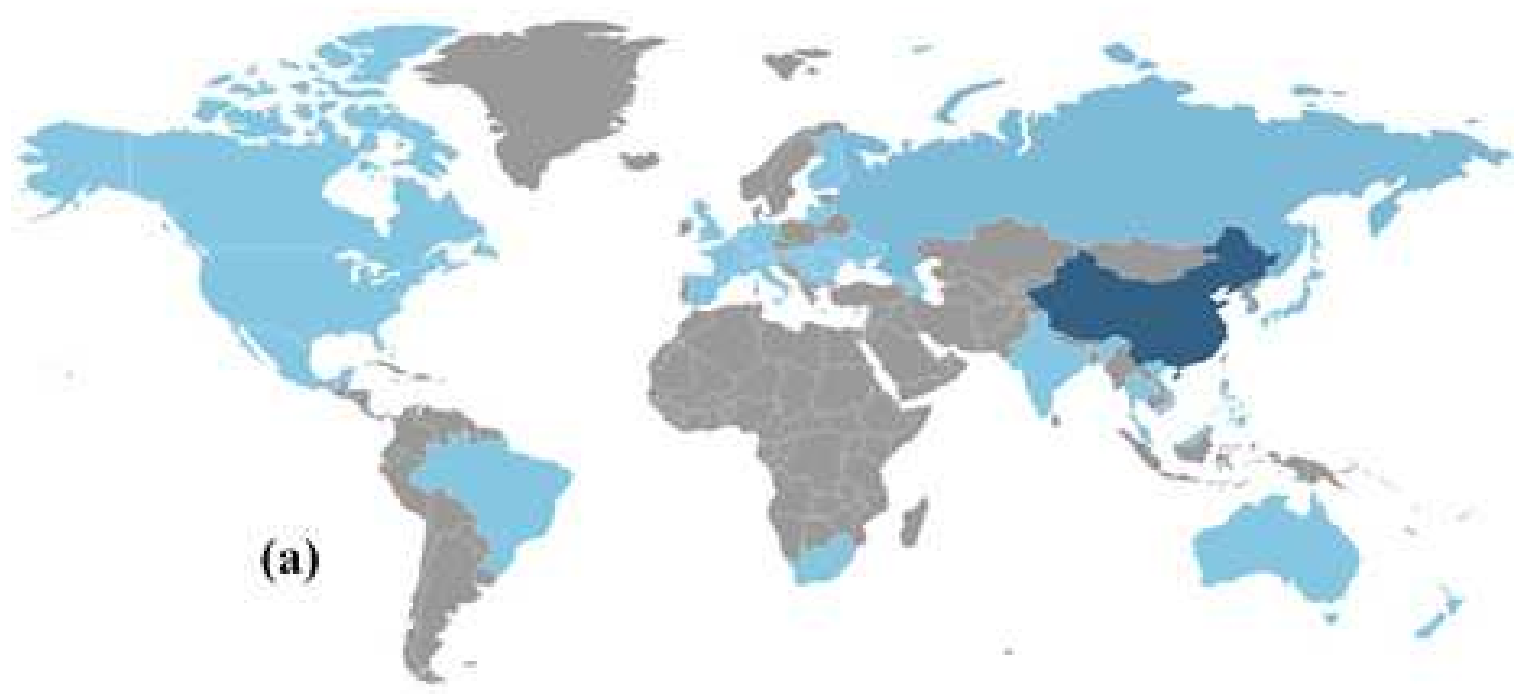

Markets a competitors location

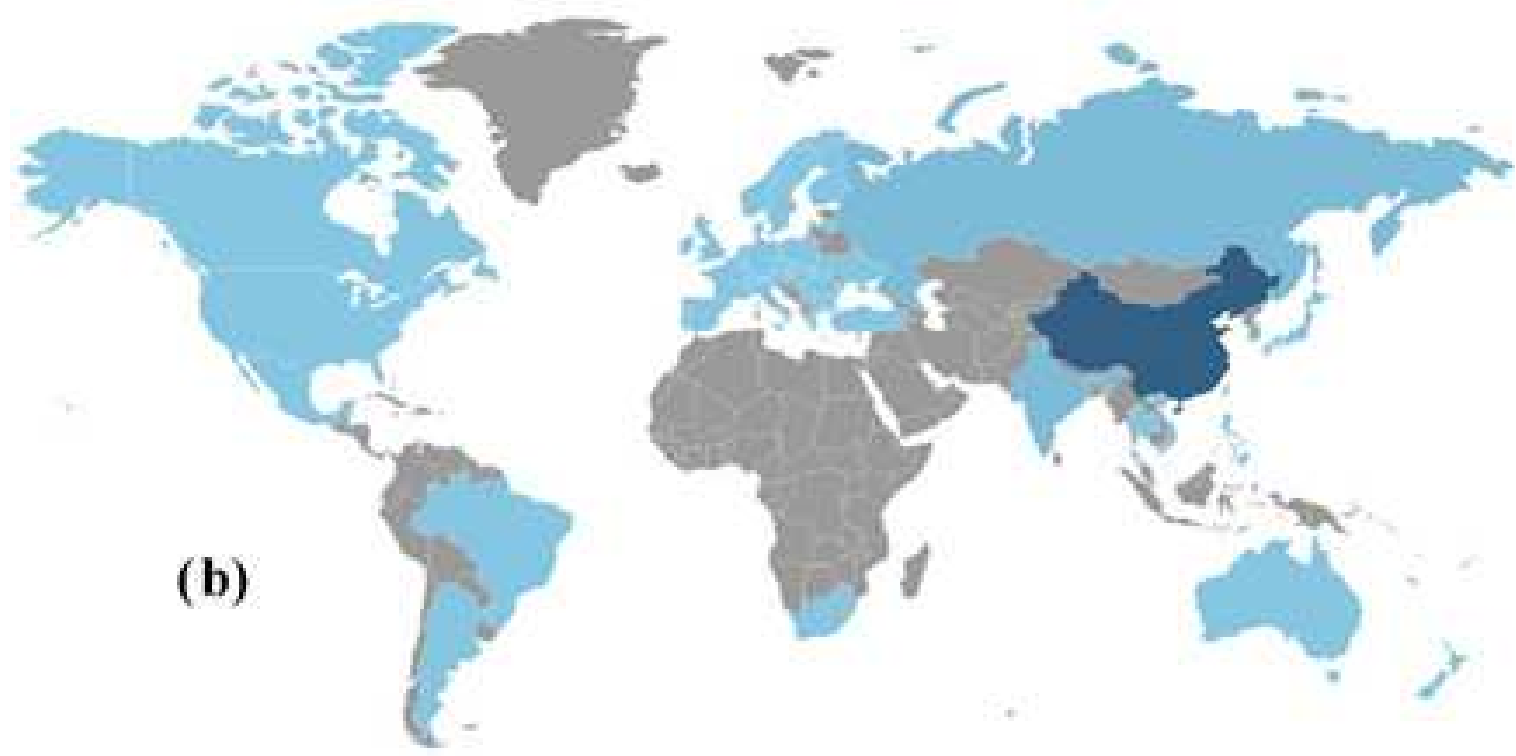

Fonte: Questel, Orbit (2018)

No contexto de citações observa-se uma repetitividade nos grupos e empresas que têm fortes interações entre si, principalmente centros de pesquisa que trabalham em colaboração. 
Estas relações existentes são importantes, mas dificultam a interação de novos setores que desejam investir na área de plantas medicinais, devido às ligações privilegiadas entre os principais "players".

\section{Considerações Finais}

Considerando-se as informações obtidas nestes estudos, é possível perceber que as plantas medicinais, bem como os fitoquímicos de um modo geral, são insumos muito pouco explorados, tanto do ponto de vista acadêmico quanto econômico, se mostrando, assim, como um campo promissor para novos empreendimentos e desenvolvimento de tecnologias, pois muito pouco ainda é realizado. Foi possível verificar que a China é o maior player nesse seguimento e que países, como a Alemanha, têm muito pouca produção, no entanto são grandes importadores de fitoquímicos. O recorte das plantas escolhidas é plenamente representativo do comportamento geral quanto ao uso da biodiversidade, seja global ou mesmo local (Brasil).

Por outro lado, o Brasil se mostra como o país que mais publica no campo das plantas medicinais, entretanto, quando se observam as tecnologias desenvolvidas por brasileiros nesse campo, os resultados são muito tímidos, mostrando a necessidade de atenção para essa área tecnológica.

Com base nessas considerações, vale ressaltar que esforços neste sentido vêm sendo despendidos, através de medidas legais, pois embora o Brasil apresente grande produção científica neste campo, muita informação é perdida pelo fato de não existir por muito tempo qualquer sistema de controle por parte de qualquer órgão relacionado ao meio ambiente que obrigasse, através de qualquer pena, pesquisadores e/ou pessoas que quisessem explorar intelectual e/ou economicamente a biodiversidade brasileira a notificar de maneira oficial seus objetivos.

Entretanto, com a nova lei da biodiversidade, Lei $n^{\circ} 13.123$, de novembro de 2015 , e do Decreto $\mathrm{n}^{\circ}$ 8.772, de 11 de maio de 2016, fica condicionada a exploração do patrimônio genético brasileiro sob qualquer forma, à condição de que se cumpra o prazo de regularização à data de disponibilização do Sistema Nacional de Gestão do Patrimônio Genético e do Conhecimento Tradicional Associado (SisGen), sob controle do Conselho de Gestão do Patrimônio Genético (CGen). Essa iniciativa pretende diminuir a burocracia antes exigida, a qual desencorajava muitos pesquisadores a oficializarem seus estudos.

Vale ressaltar que, junto ao Ministério do Meio Ambiente (MMA), outras entidades como a Associação Fórum Nacional de Gestões de Inovação (FORTEC), através de ações conjuntas, vêm promovendo treinamentos contínuos para pesquisadores e gestores de ambientes de inovação com o intuito de incentivar a boa prática relacionada à exploração do Patrimônio Genético Brasileiro, bem como à preservação do capital intelectual através da preservação $e$ identificação do conhecimento tradicional associado.

Sendo assim, esse é um campo muito promissor no mundo e, principalmente, no Brasil por possuir uma megadiversidade a ser estudada e sustentavelmente utilizada. A mudança da ótica da legislação no sentido de facilitar o registro das pesquisas, como forma de promover negócios sustentáveis, é uma aposta a ser conferida em poucos anos. 


\section{Referências}

ALBUQUERQUE, U. P.; HANAZAKI, N. As pesquisas etnodirigidas na descoberta de novos fármacos de interesse médico e farmacêutico: fragilidades e perspectivas. Revista Brasileira de Farmacognosia, v.16 (Supl.), p. 678-689, 2006.

ARNOUS A.H.; SANTOS, A.S.; BEINNER, R. P. C. Plantas medicinais de uso caseiro- conhecimento popular e interesse pelo cultivo comunitário. Espaç. Saúde, v. 6, n. 2, p. 1-6, 2005.

CORRÊA JUNIOR, C.; LIN, C.M.; SCHEFFER, M. C. SOB Informa, p. 9, 23, 1991.

MAIOLI-AZEVEDO V.; FONSECA-KRUEL V. Plantas medicinais e ritualísticas vendidas em feiras livres no município do Rio de Janeiro, RJ, Brasil: estudo de caso nas zonas Norte e Sul. Acta BotanicaBrasilica, v. 21, n. 2, p.2 63-275, 2007.

OMS. Relatório da Conferência Internacional sobre Cuidados Primários de Saúde (Alma Ata-URSS, 1978). Cuid. Primários Saúde, 1978. 64p.

SAMPAIO, E. V. S. B.; PAREYN, F. G. C.; FIGUEIROA, J. M.; SANTOS JR., A. G. Espécies do semiárido baiano com potencial econômico. Cruz das Almas, Magistra, n. 18, p. 6-8, 2006.

SIMÕES, C. M. O.; SCHENKEL, E. P.; GOSMANN, G.; DE MELLO, J. C. P.; MENTZ, L. A.; PETROVICK, P.R. (Org.). Farmacognosia: da planta ao medicamento. 4. ed. Porto Alegre: Ed. Universidade/UFRGS; Ed. da UFSC, 2002.

Farmacognosia: da planta ao medicamento. 5. ed. Porto Alegre/Florianópolis: Editora da UFSC, 2004. 1102p.

\section{Sobre as autoras}

\section{Sannyele Alcantara Emiliano}

E-mail: sannyele.ufal@gmail.com

Graduanda em Química Tecnológica e Industrial, pela Universidade Federal de Alagoas, com experiência na área de Química, com ênfase em Química Teórica, atuando principalmente nos seguintes temas: cristalografia, RM1, modelagem molecular, testes in vitro e métodos teóricos; com experiência atual na área de produtos naturais. Endereço Profissional: Universidade Federal de Alagoas, Instituto de Química e Biotecnologia - IQB. Av. Lourival de Melo Mota, S/N - Tabuleiro do Martins, Maceió - AL. CEP: 57072-970.

\section{Tatiane Luciano Balliano}

E-mail: tlb@qui.ufal.br

Graduada e Licenciada em Química pela Universidade Federal de Alagoas-UFAL (2005). Mestra em Química e Biotecnologia, área de concentração Físico-química (cristalografia de raios $\mathrm{X}$ ) pela Universidade Federal de Alagoas (2006). Doutora em Física Aplicada (Biomolecular) pela Universidade de São Paulo (2010).

Endereço Profissional: Universidade Federal de Alagoas, Instituto de Química e Biotecnologia - IQB. Av. Lourival de Melo Mota, S/N - Tabuleiro do Martins, Maceió - AL. CEP: 57072-970. 